\title{
High serum levels of CD178 (soluble FasL) predict for inferior progression free survival in chronic lymphocytic leukemia treated with fludarabine-based chemotherapy
}

\author{
Courtney Tate ${ }^{a, b}$, Melinda Burgess ${ }^{c}$, Nigel A. McMillan ${ }^{d}$, Nicholas A. Saunders ${ }^{c}$, Catherine Cheung ${ }^{c, e}$, \\ Devinder Gill ${ }^{a}$ and Peter Mollee ${ }^{a, b}$ \\ ${ }^{a}$ Department of Haematology, Princess Alexandra Hospital, Woolloongabba, Australia; ${ }^{b}$ University of Queensland, St Lucia, Australia; \\ 'Translational Research Institute, Woolloongabba, Australia; ${ }^{\mathrm{d} M e n z i e s ~ H e a l t h ~ I n s t i t u t e ~ Q u e e n s l a n d, ~ S c h o o l ~ o f ~ M e d i c a l ~ S c i e n c e s, ~ G r i f f i t h ~}$

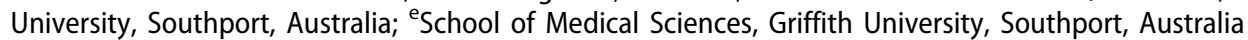

ARTICLE HISTORY Received 27 July 2018; accepted 4 February 2019

Prognosis of CLL patients has historically been determined by categorization into Rai or Binet risk groups based on physical examination and blood counts, however given heterogeneity within Rai or Binet risk categories, these prognostic tools have limitations predicting clinical course and response to therapy. More recently, the CLL-international prognostic index (CLL-IPI) discriminates four prognostic subgroups based on five independent clinical, biochemical, genetic and molecular prognostic factors including age, clinical stage, IGHV mutation status, serum $\beta 2$-microglobulin concentration and TP53 deletion and/or mutation, in an attempt to improve prognostication and to allow more targeted management of CLL patients [1].

The in vitro CLL microenvironment plays a critical role in survival of CLL cells. When CLL cells are cultured ex vivo, they undergo rapid apoptosis [2]. The Fas receptor (FasR; CD95) and Fas ligand (FasL; CD178) are cell surface proteins that belong to the tumor necrosis factor (TNF) family. Interaction of FasL with its receptor induces cell death. Soluble Fas variants are described with soluble FasR (sFasR) a product of alternative splicing, and soluble FasL (sFasL) generated by cleavage of the FasL extracellular domain by matrix metalloproteinase-family of enzymes $[3,4]$. Altered levels of soluble and membrane bound FasR and FasL have been implicated in the pathogenesis of various malignancies and autoimmune conditions. CLL cells express low levels of FasR and FasL, and high sera levels of sFasR have been associated with progressive CLL [5], however little is known about sFasL and how this impacts CLL treatment response and survival.

Therefore, we measured serum levels of 12 cytokines, including sFasL, in $32 \mathrm{CLL}$ patients participating in the German CLL Study Group CLL8 trial [6] prior to treatment with fludarabine-based therapy, compared cytokine levels in CLL patients to 7 age-matched healthy controls, and correlated cytokine levels in CLL patients to known prognostic markers and patient response rates (RR), progression free survival (PFS) and overall survival (OS). Serum CCL19, CCL21, CXCL2, CXCL12, CXCL13 concentrations were measured using commercially available ELISA kits (CCL21/6Ckine Quantikine Colorimetric ELISA (R\&D Systems), CCL19/ MIP-3b, CXCL12/SDF-1a, CXCL13/BLC/BCA-1 DuoSet ELISA Development Systems (R\&D Systems), and CXCL2/ GRO-b ELISA Development Kit (PromoKine)) according to the manufacturer's protocols. Serum CCL2, CCL3, CCL4, sCD154, sFasL, IL-6 and IL-8 concentrations were measured using BD Cytometric Bead Array Flex Sets (BD Biosciences) according to the manufacturer's protocols. Data was collected on a FACSArray Bioanalyzer cytometer (BD Biosciences) and analyzed using BD Flow Cytometric Analysis Program Array software.

Comparisons were performed using the Mann-Whitney test for two data sets, or the KruskalWallis test for greater than two data sets. Kaplan-Meier survival curves and the log-rank test were used to compare survival between CLL patients divided into two categories: those with cytokine levels above 'high' or below 'low' the median. Complete response (CR) and partial response (PR) were compared between 'high' and 'low' cytokine patient groups using the Mann-Whitney test. Cox proportional hazard regression analysis with backward elimination was used to determine multivariable independence between age ( $\leq 65$ years versus $>65$ years), clinical stage (Rai and Binet staging), IGHV status (mutation versus unmutated), serum $\beta 2$-microglobulin concentration ( $\leq 3.5 \mathrm{mg}$ / $\mathrm{L}$ versus $>3.5 \mathrm{mg} / \mathrm{L}$ ), and CLL-IPI risk groups in predicting PFS and OS. Comparison of TP53 status (no abnormalities versus deletion and/or mutation) was not performed due to insufficient numbers for statistical analysis (TP53 positive, $n=2$ ). Protocols were approved 
by the Princess Alexandra Hospital Human Ethics committee, and written informed consent was obtained from all patients according to the Declaration of Helsinki.

Table 1 shows the baseline characteristics of CLL patients. Untreated CLL patients had significantly higher CCL3, CCL4, CXCL13, sFasL and IL-6 levels, but significantly lower CXCL12 and sCD154 levels, compared with normal controls (Figure 1). Serum concentrations of CCL19, CCL21, CXCL2, CCL2 and IL-8 were no different in untreated CLL patients compared with controls.

Significantly higher levels of CCL4 were found in more advanced Rai stages (stage II-IV) and patients with higher serum 32 -microglobulin levels (Figure 2(A)), significantly higher serum levels of CCL19 were found in higher CLL-IPI risk groups (high and very high risk groups) (Figure 2(B)), and significantly higher serum levels of CCL21, CXCL13 and sFasL were found in patients with higher $\beta 2$-microglobulin levels (Figure $1(C))$. Significantly lower levels of CXCL2 were found in more advanced Rai and Binet stages, in patients with higher serum $\beta 2$-microglobulin levels and unmutated IGHV (Figure 2(D)). In addition, significantly lower levels of sCD154 were found in more advanced Rai and Binet stages (Figure 2(E)). There was no association between cytokine levels and age, sex, ECOG status, B symptoms or CIRS score (data not shown). sFasL levels did not associate with any of the currently used poor prognostic indicators.

A high sFasL serum level was associated with a lower likelihood of achieving CR (median sFasL level of
Table 1. CLL patient clinical and laboratory characteristics.

\begin{tabular}{|c|c|c|}
\hline & Number & $(\%)$ \\
\hline Total no. of subjects ${ }^{a}$ & 32 & NA \\
\hline \multicolumn{3}{|l|}{ Age, y } \\
\hline Median & 62 & NA \\
\hline Range & $40-77$ & NA \\
\hline \multicolumn{3}{|l|}{ Sex } \\
\hline Male & 27 & 84.4 \\
\hline Female & 5 & 15.6 \\
\hline \multicolumn{3}{|l|}{ Binet stage } \\
\hline B & 20 & 62.5 \\
\hline C & 12 & 37.5 \\
\hline \multicolumn{3}{|l|}{ Rai stage } \\
\hline I & 6 & 18.8 \\
\hline II & 13 & 40.6 \\
\hline III & 3 & 9.4 \\
\hline IV & 9 & 28.1 \\
\hline \multicolumn{3}{|l|}{ IGHV mutation status } \\
\hline Mutated & 13 & 40.6 \\
\hline Unmutated & 17 & 53.1 \\
\hline \multicolumn{3}{|l|}{$\beta 2$-microglobulin } \\
\hline$\leq 3.5 \mathrm{mg} / \mathrm{L}$ & 21 & 65.6 \\
\hline$>3.5 \mathrm{mg} / \mathrm{L}$ & 10 & 31.3 \\
\hline \multicolumn{3}{|l|}{ Fluorescent in situ hybridization(FISH) } \\
\hline $17 p$ deletion & 2 & 6.3 \\
\hline $11 q$ deletion (no $17 p$ deletion) & 4 & 12.5 \\
\hline $12 q$ trisomy (no $17 p$ or $11 q$ deletion) & 3 & 9.4 \\
\hline Normal & 7 & 21.9 \\
\hline 13q deletion as sole abberation & 11 & 34.4 \\
\hline \multicolumn{3}{|l|}{ CLL-IPI risk group } \\
\hline Low & 5 & 15.6 \\
\hline Intermediate & 8 & 25.0 \\
\hline High & 12 & 37.5 \\
\hline Very high & 2 & 6.3 \\
\hline \multicolumn{3}{|l|}{ Treatment } \\
\hline Fludarabine and cyclophosphamide (FC) & 17 & 53.0 \\
\hline FC plus rituximab (FCR) & 15 & 47.0 \\
\hline
\end{tabular}

${ }^{a}$ Clinical data were not available on all 32 CLL patients: Rai stage and $\beta 2$ microglobulin (31/32), IGHV mutation status (30/32), FISH and CLL-IPI (27/32). NA indicates not applicable.
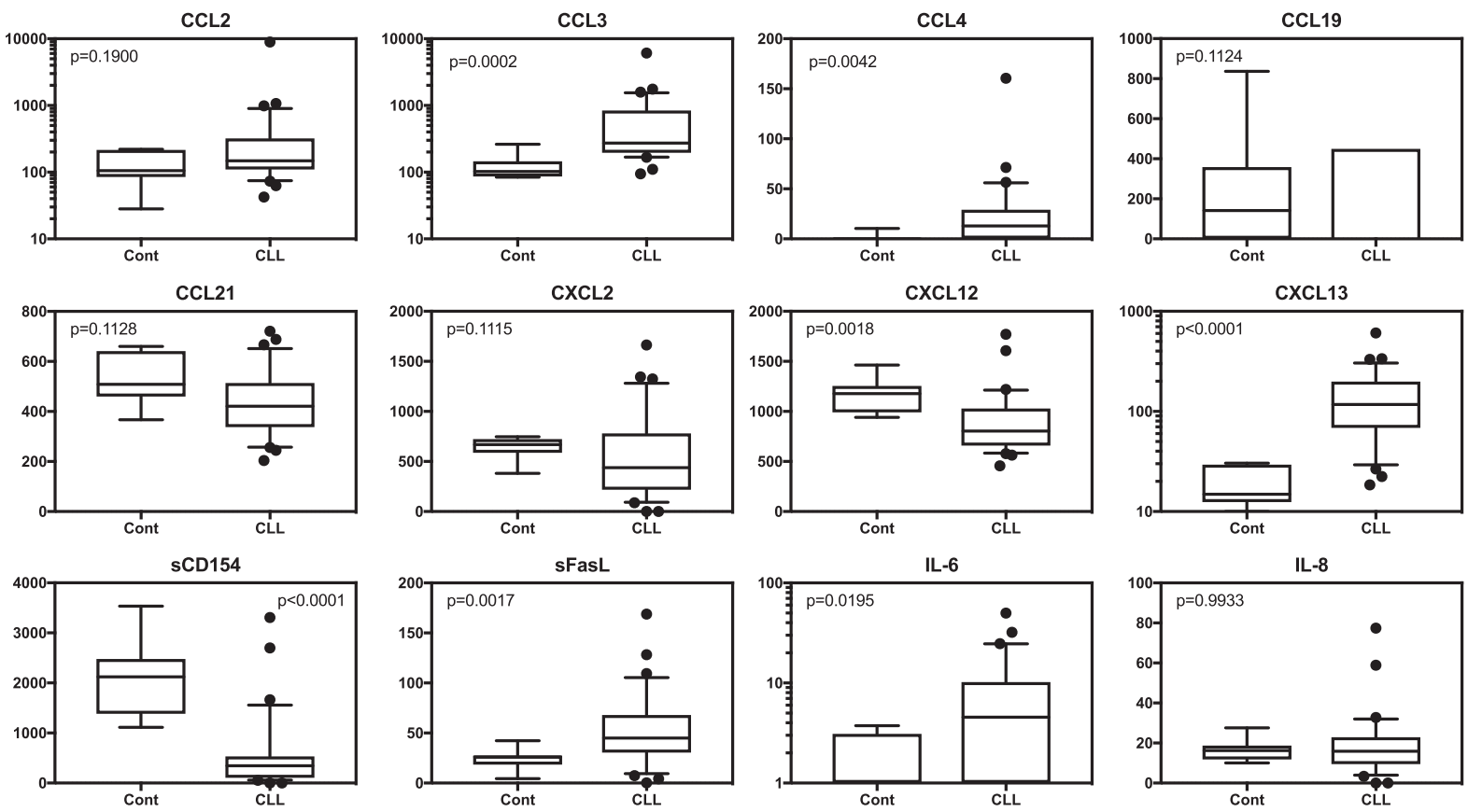

Figure 1. Differential expression of cytokines in untreated CLL patients compared with age-matched healthy controls. The bottom and top of the box plots indicate the 25th and 75th percentiles respectively, the bar within the box indicates the median value, and the ends of the whiskers indicate the 10th and 90th percentiles. Outliers are represented by dots. Cytokine levels are reported in picograms/milliliter. Note CCL2, CCL3, CXCL13, IL-6 cytokine levels are represented graphically using a log10 scale on the y-axis. All other cytokine levels are represented using a linear scale. 
(A)

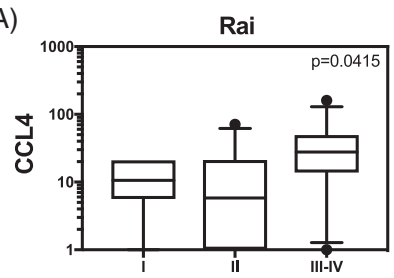

(C)

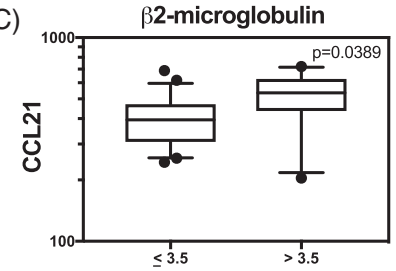

(D)

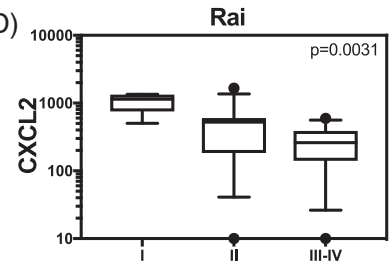

(E)

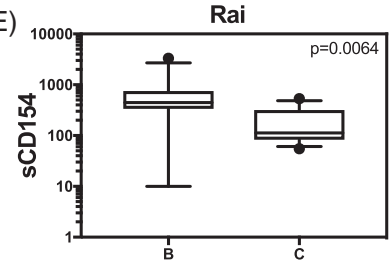

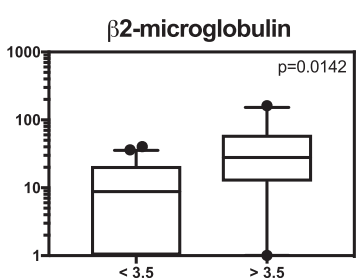

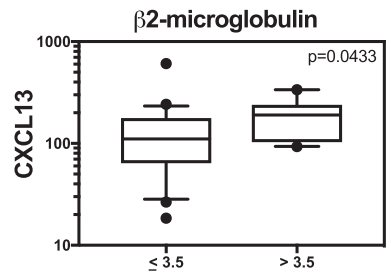

Binet

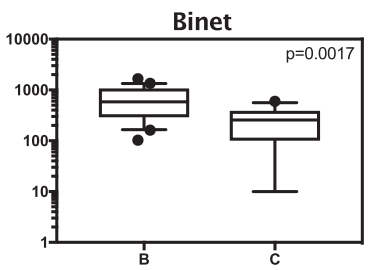

Binet

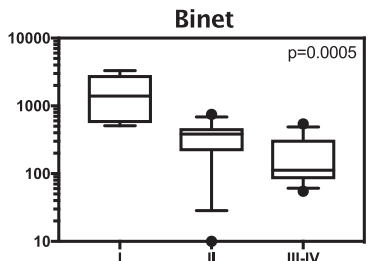

(B)

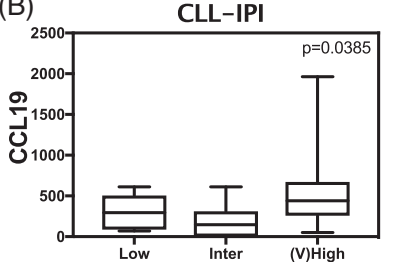

$\beta 2$-microglobulin

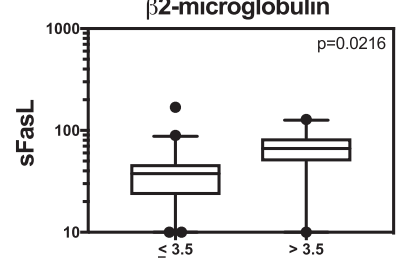

$\beta 2$-microglobulin
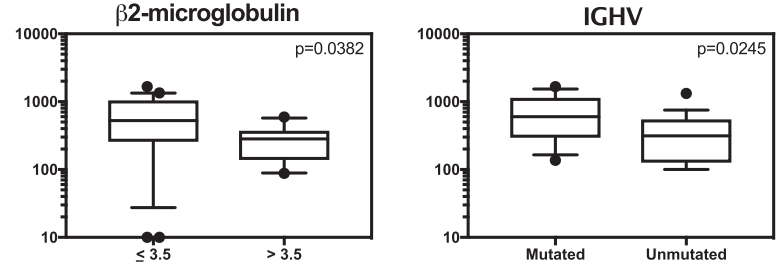

Figure 2. Cytokines for which serum levels are significantly different when untreated CLL patients are analyzed according to Rai and Binet staging, serum 32 -microglobulin levels, IGHV mutation status and CLL-IPI risk groups. Data are presented as box plots (see Figure 1 for details). To allow statistical analysis of low absolute numbers, Binet stage III-IV and CLL-IPI high risk and very high risk are grouped together [(V) High]. Serum 32 -microglobulin levels are reported in milligrams/litre ( $x$-axis). Cytokine levels are reported in picograms/milliliter (y-axis). Note all cytokine levels are represented graphically using a log10 scale on the y-axis, except CCL19, which is represented using a linear scale.

$30.35 \mathrm{pg} / \mathrm{ml}$ (range 4.05-39.5) in patients achieving CR versus $46.48 \mathrm{pg} / \mathrm{ml}$ (range $0-128.2$ ) in patients achieving PR). In addition, a high sFasL predicted for inferior PFS ( $72 \%$ for low sFasL versus $20 \%$ for high sFasL at 48 months, Figure 3(B)). This effect on PFS was independent of Binet and Rai stages, IGHV mutation status, serum $\beta 2$ microglobulin concentration, CLL-IPI score and whether patients received fludarabine and cyclophosphamide (FC), or FC plus rituximab (FCR) (Figure $3(\mathrm{~A}-\mathrm{C})$ ). Only the CLLIPI score was an independent prognostic marker for OS $(\mathrm{HR}=5.214 ; 95 \% \mathrm{Cl} 1.370-19.847, p=.015)$. This was despite an OS of low sFasL versus high sFasL patients being $85 \%$ versus $60 \%$ at 48 months, and $78 \%$ versus $53 \%$ at 60 months (Figure 3(D)).

In the current era of novel targeted agents, FCR remains standard therapy for younger patients with CLL, particularly for those with mutated IGHV gene status and no TP53 deletion [7]. Markers that can predict which patients are unlikely to respond to fludarabine-based therapy would be useful given the significant treatmentassociated morbidity. To our knowledge, this is the first demonstration of lower levels of SCD154 and CXCL12 in
CLL sera, and that lower levels of SCD154 and CXCL2 and higher levels of CLL4, CCL19, CCL21, and sFasL correlate with poor prognostic factors, but that only sFasL predicts for lower CR rates and inferior PFS to fludarabine-based chemotherapy. Our findings of elevated CCL3, CCL4, CXCL13, IL-6 and SFasL sera levels are consistent with previous reports [8-12].

The mechanism by which sFasL results in lower CR and inferior PFS in CLL patients treated with fludarabinebased chemotherapy is unknown, however abnormal levels of SFasL have been detected in sera from patients with B- and T-cell malignancies, solid tumors, and patients with autoimmune disorders [12-14], and high levels of sFasL in patients with large granulocytic leukemia (LGL) has been shown to protect the leukemic LGLs from apoptosis in vivo [15].

Limitations of this study include preliminary results in a small number of patients and the need to validate in a larger cohort. In addition, feasibility of FasL testing in the clinical setting is uncertain. Future studies may be directed at determining how TP53 mutations correlate with SFasL levels (TP53 sequencing was not performed in 


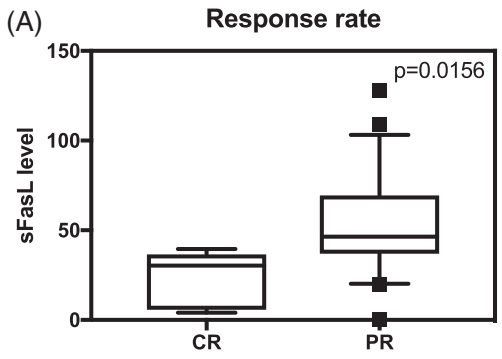

(C) PFS depending on treatment received

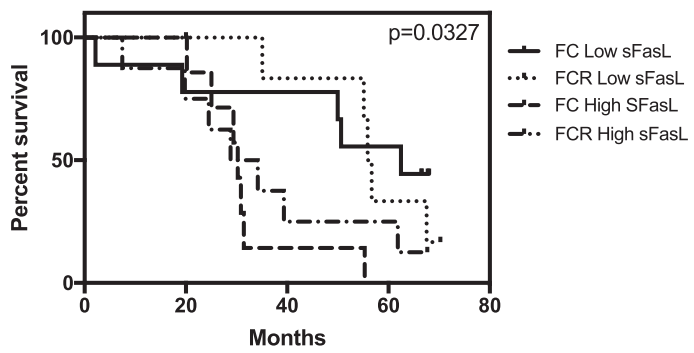

(B) Pogression Free Survival

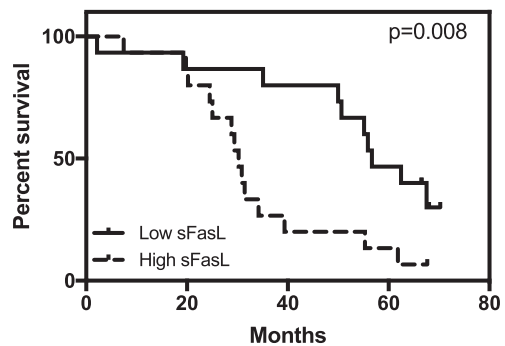

(D)

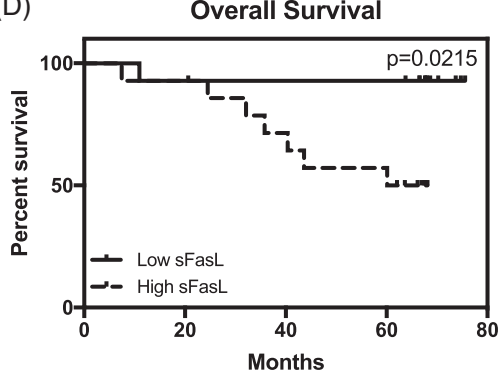

Figure 3. Higher pretreatment serum sFasL concentration predicts for lower complete response (CR) rates and inferior progression free survival (PFS). (A) Serum sFasL levels pretreatment are lower in those that achieve a CR compared to a partial response (PR). Data are represented as box plots (see Figure 1 for details). Serum sFasL levels are reported in picograms per milliliter. (B-D) Kaplan-Meier analysis shows higher levels of sFasL correlate with shorter PFS (54 months vs 34 months; $p=.008 ; \mathrm{HR} 3.2 ; 95 \% \mathrm{Cl}$ 1.4-7.8). Length of PFS was independent of whether fludarabine and cyclophosphamide (FC) or FC plus rituximab (FCR) was received (62.5 (FC; low sFasL) or 56.3 (FCR; low sFasL) months vs 30.2 (FC; high sFasL) or 31.5 (FCR; high sFasL) months; $p=.0327$ ). The difference in overall survival failed to reach statistical significance (68 months vs 53 months; $p=.183 ; \mathrm{HR} 2.5 ; 95 \%$ $\mathrm{Cl}$ 0.6-9.7).

the CLL8 cohort), or whether sFasL can predict patient response to novel agents such as kinase inhibitors of the B-cell receptor pathway and BCL2 antagonists. Furthermore, if future functional studies determine sFasL has a pathological role in CLL biology, this may provide a potential therapeutic target in CLL.

In conclusion, we show that sFasL predicts for lower $C R$ rates and inferior PFS in CLL patients treated with fludarabine-based therapy. Combining sFasL with previous prognostic tools may result in refined algorithms that are able to predict response to various therapies, allowing for targeted treatment strategies.

\section{Acknowledgements}

The authors would like to thank Professor Michael Hallek and Mr. Justin Scott.

Potential conflict of interest: Disclosure forms provided by the authors are available with the full text of this article online at https://doi.org/10.1080/10428194.2019.1581936.

\section{Funding}

This work was supported by a Leukaemia Foundation Australia Grant-In-Aid 2010.

\section{References}

[1] International CLL-IPI working group. An international prognostic index for patients with chronic lymphocytic leukaemia (CLL-IPI): a meta-analysis of individual patient data. Lancet Oncol. 2016;17:779-790.

[2] Collins RJ, Verschuer LA, Harmon BV, et al. Spontaneous programmed death (apoptosis) of B-chronic lymphocytic leukaemia cells following their culture in vitro. Br J Haematol. 1989; 71:343-350.

[3] Ayroldi E, D'Adamio F, Zollo O, et al. Cloning and expression of a short Fas ligand: a new alternatively spliced product of the mouse Fas ligand gene. Blood. 1999;94:3456-3467.

[4] Kayagaki N, Kawasaki A, Ebata T, et al. Metalloproteinasemediated release of human Fas ligand. J Exp Med. 1995;182: 1777-1783.

[5] Osorio LM, Aguilar-Santelises M, De Santiago A, et al. Increased serum levels of soluble Fas in progressive B-CLL. Eur J Haematol. 2001;66:342-346.

[6] Hallek M, Fischer K, Fingerle-Rowson G, et al. International Group of Investigators; German Chronic Lymphocytic Leukaemia Study Group. Addition of rituximab to fludarabine and cyclophosphamide in patients with chronic lymphocytic leukaemia: a randomised, open- label, phase 3 trial. Lancet. 2010;376:1164-1174.

[7] Chai-Adisaksopha C, Brown JR. FCR achieves long-term durable remissions in patients with IGHV-mutated CLL. Blood. 2017;130:2278-2282.

[8] Yan XJ, Dozmorov I, Li W, et al. Identification of outcomecorrelated cytokine clusters in chronic lymphocytic leukemia. Blood. 2011;118:5201-5210.

[9] Fayad L, Keating MJ, Reuben JM, et al. Interleukin-6 and interleukin-10 levels in chronic lymphocytic leukemia: 
correlation with phenotypic characteristics and outcome. Blood. 2001;97:256-263.

[10] Anand M, Chodda SK, Parikh PM, et al. Dysregulated cytokine production by monocytes from chronic lymphocytic leukemia patients. Cancer Biother Radiopharm. 1998;13:43-48.

[11] Sivina M, Hartmann E, Kipps TJ, et al. CCL3 (MIP-1\{alpha\}) plasma levels and the risk for disease progression in chronic lymphocytic leukemia (CLL). Blood. 2011;117:1662-1669.

[12] Knipping E, Debatin KM, Stricker K, et al. Identification of soluble APO-1 in supernatants of human B- and T-cell lines and increased serum levels in B- and T-cell leukemias. Blood. 1995;85:1562-1569.

[13] Knipping E, Krammer $\mathrm{PH}$, Onel $\mathrm{KB}$, et al. Levels of soluble Fas/APO-1/CD95 in systemic lupus erythematosus and juvenile rheumatoid arthritis. Arthritis Rheum. 1995;38:1735-1737.

[14] Peter ME, Hadji A, Murmann AE, et al. The role of CD95 and CD95 ligand in cancer. Cell Death Differ. 2015;22:549-559.

[15] Liu JH, Wei S, Lamy $\mathrm{T}$, et al. Blockade of Fas-dependent apoptosis by soluble Fas in LGL leukemia. Blood. 2002;100: 1449-1453. 